\title{
Differential Expression Profiles of Mitogenome Associated MicroRNAs Among Colorectal Adenomatous Polyps
}

\author{
LaShanale Wallace $^{1}$, Karen Aikhionbare ${ }^{2}$, Saswati Banerjee ${ }^{3}$, Katie Peagler ${ }^{1}$, Mareena Pitts ${ }^{1}$, \\ Xuebiao $\mathrm{Yao}^{3}$, Felix Aikhionbare ${ }^{1, \text {, }}$ \\ ${ }^{1}$ Department of Medicine, Morehouse School of Medicine, Atlanta, Georgia, USA \\ ${ }^{2}$ College of Science and Mathematics, Augusta University, Augusta, Georgia, USA \\ ${ }^{3}$ Department of Physiology, Morehouse School of Medicine, Atlanta, Georgia, USA \\ Email address: \\ nelliew9@gmail.com (L. Wallace), kaikhionbare@augusta.edu (K. Aikhionbare), sbanerjee@msm.edu (S. Banerjee), \\ kpeagler@msm.edu (K. Peagler), mpitts@msm.edu (M. Pitts), xyao@msm.edu (Xuebiao Yao), faikhionbare@msm.edu (F. Aikhionbare) \\ ${ }^{*}$ Corresponding author
}

\section{To cite this article:}

LaShanale Wallace, Karen Aikhionbare, Saswati Banerjee, Katie Peagler, Mareena Pitts, Xuebiao Yao, Felix Aikhionbare. Differential Expression Profiles of Mitogenome Associated MicroRNAs Among Colorectal Adenomatous Polyps. Cancer Research Journal.

Vol. 9, No. 1, 2021, pp. 23-33. doi: 10.11648/j.crj.20210901.14

Received: January 1, 2021; Accepted: January 19, 2021; Published: January 25, 2021

\begin{abstract}
Colorectal tumors are mostly of epithelial origin and represent a wide spectrum of neoplasms. About $97 \%$ of colorectal cancer originating from benign lesions of adenomatous polyps are adenocarcinomas. Reactive oxygen species (ROS) generating from mitochondrial DNA (mtDNA) mutations and microRNAs (miRNAs) are associated with oncogene and tumor suppressor genes regulation which are known to parallel the tissue abnormalities involved with tumorigenesis such as colorectal adenoma to adenocarcinoma. However, the differential expression patterns of mitochondrial associated microRNAs (referred as MitomiRs) among colorectal adenomatous polyps progression is yet to be determined. Thus, the aim of this study was to determine the differential expressions profiles of MitomiRs (miR-24, miR-181, miR-210, miR-21 and miR378) in patients with colorectal adenomatous polyps tissues in correlation with clinicopathological tumor architectures of tubular, tubulovillous, villous adenomas and adenocarcinomas. Isolation of mitochondria RNA from colorectal adenomatous polyps, adenocarcinomas, and normal adjacent tissue samples was performed and assessed for mitochondrial associated miRNAs expression differences using quantitative reverse transcription PCR. Data from this study demonstrates that mitochondria genome expression of mitomiRNAs; miR-24, miR-181, miR-210, miR-21 and miR-378 in colorectal tissue samples varies among the adenomatous polyps. Expression of mitomiRNAs 24, 181, 210 and 378 progressively increased from the precancerous of adenomatous polyps to adenocarcinoma. In addition, miR-210 and miR-181 expression increased 3 folds in villous adenomas and greater than 3 folds increased in miR378 in adenocarcinoma $(p<0.005)$ when compared to tubular adenoma. Meanwhile, miR-21 increased progressively in adenoma tissues but decreased almost 2.5 folds in adenocarcinomas when compared to villous adenoma tissues $(p<0.001$ ). These results suggest mitomiRs may regulate important mitochondrial functional pathways leading to a more favorable environment for transformation or progression of colorectal adenomatous polyps into adenocarcinomas.
\end{abstract}

Keywords: Colorectal Adenomas, Mitochondrial microRNA (Denoted: mitomiRNAs), Reactive Oxygen Species, CRC Tissues

\section{Introduction}

Colorectal cancer (CRC) is the third leading cause of cancer related deaths in both men and women in the United States [1]. Survival rates are closely related to the stage of
$\mathrm{CRC}$ at the time of diagnosis $[1,2]$. Colorectal tumors are mostly of epithelial origin that represent a wide spectrum of neoplasms. About $97 \%$ of colorectal cancer originating from benign lesions of adenomatous polyps are adenocarcinomas [2]. The best evaluation of colorectal tumor progression, 
especially the early adenomas, continues to confound clinicians and the presence of colorectal adenomas dysplasia subtype, increases one's risk of developing colorectal cancer [2]. There is a pressing need to gain more insights into the biology of colorectal adenomatous polyps to facilitate the early detection and for therapeutic measures. Several lines of evidence have suggested that $\mathrm{CRC}$ is a result of multiple mutations in both tumor suppressor genes $\left(\mathrm{P}^{53}, \mathrm{DCC}, \mathrm{APC}\right.$, $\mathrm{P}^{27}$ ) and oncogenes (KRAS, EGFR, TGF alfa) which are key players in cell growth regulation [3-5]. Past studies have shown that miRNAs post transcriptionally regulated gene expression by binding to the 3' untranslated regions of target messenger RNAs and decrease or stop their translation to proteins [6]. Moreover, a variety of molecular pathways associated with cancer and various other diseases have been attributed to miRNAs regulation [7, 8]. Recently, it was suggested that pre-miRNAs as well as mature miRNAs are present in the mitochondria refer to as mitomiRs. Some of these processed pre-miRNAs sequences are actively involved in mitochondrial transcripts or possibly exported in the cytosol to interfere with its genomic mRNA [8]. Alterations in miRNA expression have been described previously in CRC creating a unique area for an alternative diagnostic approach [9]. In addition multiple studies have reported that miR-18a-5p, miR-21-5p, miR-29a-5p, miR-92a-5p, miR143-5p and miR-378-5p are the most dysregulated circulating miRNAs in CRC $[7,8,10]$ and many of these relationships have explored in the involvement of miRNAs on CRC mutated genes, such as TP53 [11], APC [12], and KRAS [4, 5]. Studies have indicated that miR-21 expression increases as tumor stages progress and, also miR-21 including miR210 and mi181 have been linked to the regulation of reactive oxygen species (ROS) in relevant to the clinical significance in CRC [9, 13-16]. ROS are primarily generated during mitochondrial respiration and both mtDNA and protein are susceptible to insults from ROS which are produced during oxidative phosphorylation [17]. Previous studies have demonstrated that certain mtDNA mutations as results of ROS damages are associated with colorectal adenomas progression $[18,19]$. Additionally, it was suggested that there are changes in the expression of mitochondrial genes as indicated by the progressive increased in carbonyl content from early colorectal adenomas to adenocarcinomas. Given that $\mathrm{CRC}$ is an age-related disease and accumulation of dysfunctional mitochondria during aging can therefore generate a considerable amount of ROS and oxidative stress. Understanding microRNAs associated with mitochondria (mitomiRs) regulation of protein-coding gene expression patterns may perhaps help clarify how these diverse genetic pathways of colorectal adenomas dysplasia subtypes are connected and regulated. In this study we determined the differential expression profiles of mitomiRs of 24, 181, 210, 21 and 378 among patient adenomatous polyp tissues of CRC in correlation within clinicopathological epithelial tumor architectures of tubular, tubulovillous, villous adenomas and adenocarcinomas. These selected miRNAs in this study have been previously established as a tumor suppressor or oncogene in epithelial cancer types [10, 13, 20, 21, 22].

\section{Materials and Methods}

\subsection{Tissue Samples}

Table 1. Clinical data for 64 pairs of the colorectal tumor samples.

\begin{tabular}{ll}
\hline Variable & Cases \\
\hline Age, (Years) & $\mathrm{n}^{* *}$ \\
Above 50 & 48 \\
Below 50 & 16 \\
Gender & \\
Female & 40 \\
Male & 24 \\
Histological Stage & \\
NormalAdjacent/TumorTissue (Pairs) & \\
Tubular Adenoma & 16 \\
Tubulovillous Adenoma & 16 \\
Villous Adenoma & 16 \\
Adenocarcinoma Pooled* & 16 \\
Adenocarcinoma Pooled Breakdown* & \\
Stage I & 4 \\
Stage II & 4 \\
Stage III & 4 \\
Stage IV & 4 \\
\hline
\end{tabular}

$\mathrm{n}^{* *}$ Sample tissue size: Tumors and normal surrounding tissue samples are in pairs.

*Pooled of stages (I-IV) and their normal adjacent tissues referred as adenocarcinomas in this study based on American Joint Cancer Committee criteria.

Sixty-four pairs of tissues utilized in this study were preexisting coded samples that were not involved in any activity that alters therapy or patient management. The study samples were directly from the primary archival of patient tissues who underwent routine surgical resections through the Cooperative Human Tissue Network of the southern region. Protocols of these studies were implemented by the approval of both Institutional Review Boards of Morehouse School of Medicine and the University of Alabama at Birmingham. Familial adenomatous polyposis, hereditary nonpolyposis syndrome, or inflammatory bowel disease patient tissue samples were excluded from this study. Inclusion selected samples were adjacent normal $(\mathrm{N})$ tissues obtained from adenomatous polyps which comprise of tubular (TA), tubulovillous (TV), villous (V) adenomas, and invasive carcinomas, which were graded as adenocarcinoma (CA) of pooled stages I-IV which are based on the degree of their glandular differentiations. Stage I adenocarcinomas were defined as well-differentiated, more than $85 \%$ of tumors composed of glandular adenocarcinomas; moderately differentiated adenocarcinomas within 50\%-95\% composed of glandular tumors were defined as stages II; while stages III were defined as poorly differentiated carcinomas within $49 \%$ or less composed of glandular tumors; stages IV were poorly differentiated carcinomas with more $50 \%$ composed of glandular tumors. Studied tissue samples were microdissected to excise adjacent normal tissues from an area at least $5 \mathrm{~cm}$ away from the tumors. Diagnosis and histopathologically staging of all tumor samples in this study 
were performed by pathologists based on the seventh edition Tumor-Node-Metastasis (TNM) staging system of the American Joint Committee on Cancer (AJCC). The demographics of 64 pairs of frozen primary CRC tissues (including tumors and their adjacent normal controls) in this study are shown in Table 1. The breakdown of the 64 pairs of tissues were as follows: N/TA $(n=16), N / T V(n=16), N / V$ $(n=16)$, and N/CA $(n=16)$ as shown in Table 1 . Of the 64 paired tissue samples, 24 were from African Americans (AA), 38 were from Caucasians (CAU), and two were unknown demographics. Twenty-six of the sample pairs were from men and 38 from women, with the median age of 72 .

\subsection{Mitochondrial Genome Isolation}

All the 64 pairs tissue specimens were micro-dissected with the use of an Arctus PixCell II microscope (Arcturus Engineering) to increase the quality and for replication of the distance excised at least $5 \mathrm{~cm}$ between the colorectal tumors and matched surrounding precancerous normal-appearing cells. Additionally, tissue samples were histologically staged by pathologist based on criteria of the AJCC. Isolation of whole mitochondrial populations from each tissue samples were performed using BioVision isolation kits according to the manufacturer's protocols (BioVision, Research Products, Milpitas, CA US). Quality of the isolated whole mitochondria and the integrity of membrane were assessed using the Mitochondria Staining Kit of JC-1 test (CS0390, Sigma, US). This test is based on electrochemical proton gradient of mitochondrial inner membrane. Stabilization of the extracted whole mitochondrial samples were done by snap-freezing immediately in cryovials and immersed in liquid nitrogen after excision and dissection. For long-term storage, the extracted tissue samples were transferred to $80^{\circ} \mathrm{C}$ freezer as recommended for the measurement of RNA and proteins.

Table 2. Candidate MicroRNA Sequences.

\begin{tabular}{|c|c|c|}
\hline MicroRNA & Mature Sequence & Sequence \\
\hline hsa-mir-210-3p & $\begin{array}{l}\text { ACCCGGCAGUGCCUCCAGGCGCAGGGCAGCCCCUGCCCACCGCACACUGCGCUGCC } \\
\text { CCAGACCCACUGUGCGUGUGACAGCGGCUGAUCUGUGCCUGGGCAGCGCGACCC }\end{array}$ & CUGUGCGUGUGACAGCGGCUGA \\
\hline hsa-mir-21-5p & $\begin{array}{l}\text { UGUCGGGUAGCUUAUCAGACUGAUGUUGACUGUUGAAUCUCAUGGCAACACCAGU } \\
\text { CGAUGGGCUGUCUGACA }\end{array}$ & UAGCUUAUCAGACUGAUGUUGA \\
\hline hsa-mir-24-3p & $\begin{array}{l}\text { CUCCGGUGCCUACUGAGCUGAUAUCAGUUCUCAUUUUACACACUGGCUCAGUUCA } \\
\text { GCAGGAACAGGAG }\end{array}$ & UGGCUCAGUUCAGCAGGAACAG \\
\hline hsa-mir-181c-3p & $\begin{array}{l}\text { CGGAAAAUUUGCCAAGGGUUUGGGGGAACAUUCAACCUGUCGGUGAGUUUGGGCA } \\
\text { GCUCAGGCAAACCAUCGACCGUUGAGUGGACCCUGAGGCCUGGAAUUGCCAUCCU }\end{array}$ & AACCAUCGACCGUUGAGUGGAC \\
\hline hsa-mir-103a-5p & $\begin{array}{l}\text { UACUGCCCUCGGCUUCUUUACAGUGCUGCCUUGUUGCAUAUGGAUCAAGCAGCAU } \\
\text { UGUACAGGGCUAUGAAGGCAUUG }\end{array}$ & AGCAGCAUUGUACAGGGCUAUGA \\
\hline hsa-mir-378-5p & $\begin{array}{l}\text { GGUCAUUGAGUCUUCAAGGCUAGUGGAAAGAGCACUGGACUUGGAGGCAGAAAG } \\
\text { ACC }\end{array}$ & ACUGGACUUGGAGGCAGAA \\
\hline
\end{tabular}

\subsection{RNA extraction and Quantitative-PCR Analysis}

Extraction of total RNA from tissue samples were performed with RNeasyMini kits (Qiagen, Germantown, MD, US). Briefly, Frozen whole mitochondrial samples were homogenized on ice and washed with PBS twice following the manufacturer's protocol. To reduce viscosity, samples were placed in QIAshredder (Qiagen, Germantown, MD, US) column, after tissue homogenization. Purification of the RNA was assessed by $1 \%$ agarose gel electrophoresis and quantified with a Nanodrop ND-2000 spectrophotometer (Nanodrop Technologies, Wilmington, DE, US). RNA concentrations were adjusted to equal concentrations prior to analysis for complementary DNA (cDNA) polymerization. Following the manufacturer's instructions using $500 \mathrm{ng}$ of total RNA as a template and random hexamers as primers, reverse transcription (RT) PCR was performed with iscript cDNA synthesis kits (BioRad, Hercules, CA, US). Quantitative PCR was performed in a 480 Light cycler detector (Roche Diagnostics, Indianapolis, IN, US) with a SYBR green I master mix, $10 \mathrm{ng}$ of $\mathrm{cDNA}$, and with designed specific primers of mitomiRNAs 24, 181, 210, 21 and 378 (Table 2). Each miRNA primer was normalized with miR-103a-3p and with adjacent normal and tumor tissue samples as previously demonstrated by Heidi and Latham [23]. These five miRNA sequences (miR-24, miR-181,
miR-210, miR-21 and miR-378) were particularly chosen based on previously published data on epithelial cancer types $[10,13,20-22]$. These selected miRNAs have been established as a tumor suppressor or an oncogene according to Sanger Center miRNA Registry at http://www.sanger.ac.uk/Software/ Rfam/mirna/index.shtml. The biological targets prediction of the selected miRNAs and validation of the microRNA-target interactions both in vitro and in vivo were done using the databases (miRDB, TargetScan, miRBase and miRTarBase) as previously described [20]. For confirmation of primers specificity, 1\% agarose gel electrophoresis was utilized to check for the expected single product length for each RT-qPCR transcript. The standard curves for each primer sets were generated using serial dilutions of pooled cDNA to standardize variations in PCR reactions as we have previously published in our lab [Wallace et al. 24]. To verify the melting temperature of the amplicons after each run, melting curve were utilized and all qPCR reactions were performed in triplicates. To calculate the crossing point $(\mathrm{Cp})$ values and the mRNA concentrations, second-derivative maximum analysis was used. For further calculations of each gene, the value means of the three replicates for each sample were used and RNA expression level ratios in tumor tissues were based on comparisons of tumors and their adjacent normal tissues as previously described [20]. Calculations of DCt were done by 
subtracting the $\mathrm{Ct}$ values of internal controls from the $\mathrm{Ct}$ values of the miRNAs of interest, and mean DCt values were compared between CRC precancerous adenomas and normal adjacent controls.

\subsection{Statistical Analysis}

The expression levels of miRNA were compared between CRC precancerous adenomas of tubular, tubulovillous, villous and between advanced adenomas (referred as adenocarcinoma) (stages I-IV) and the surrounding normal as controls using the Wilcoxon- Mann-Whitney-Test (hereafter: ANOVA test). All test values were reported as the means \pm the standard error. A one-way analysis of variants (ANOVA) was used to assess the differences using GraphPad Prism 7 software. Statistical significance was considered at $\mathrm{p}$ value of $P<0.05$.

\section{Results}

The total RNA extracted from each tissue was used to measure the expression levels of mitomiRs-24, 181, 210, 21 and 378 in CRC precancerous adenomas and stages of adenocarcinoma (CA).

In figure1A, mitomiR-24 expression levels were varied between pooled adenocarcinoma and all adenoma types of tubular, tubulovillous and villous. There was a minimal increased in the expressions of miR-24 between the earlier adenomas and late adenoma tissues with significantly increased in pooled adenocarcinoma (CA) $(p<0.001)$. However, analysis of two individual patient samples with difference in CRC stages of various degrees of tumor cells showed that expression levels of mitomiR-24 in adenocarcinoma and late precancerous tissues of villous adenoma were relatively based on the number of tumor cells in each tissue and the stage as shown in Figures 1B and 1C.

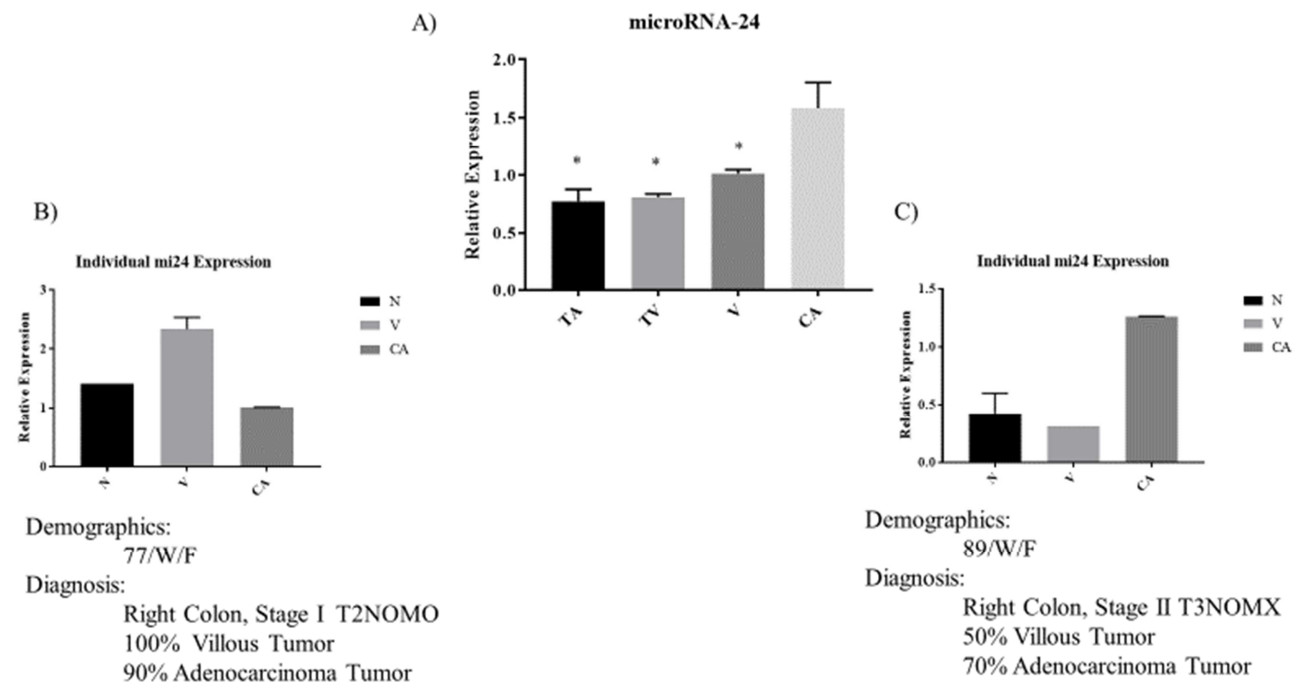

Figure 1. The relative expression levels of MitomiRNA-24 observed among patient tissue samples ( $n=64$ pairs) of colorectal adenomas (tubular (T), tubulovillous (TV), villous (V)), pooled adenocarcinoma (CA) and their normal adjacent tissues (N). A) Correlation of each clinicopathological features of colorectal adenomas and pooled adenocarcinoma tissues with expression levels of mitomiRNA-24 in extracted mitochondria RNA. Mean expression values from each tissue histological type were pooled and normalized to their paired normal adjacent tissues. Values are presented as relative expressions of mitomiRNA-24 which showed markedly increased progressively from colorectal adenomas to the pooled adenocarcinomas. B) Comparison of mitomiRNA-24 expression patterns in normal adjacent, villous, and adenoma tissues based on individual patient. Patient one is defined as 77 years old white female with stage I colon cancer. C) Comparison of mitomiRNA-24 expression patterns in 89 years old white female with stage II colon cancer. Bar diagrams show mean \pm from pooled results. Asterisk (") $P<0.005$.

We observed significant expression levels of mitomiR-181 with progressively increased in the sample tissues of CRC from precancerous of tubular adenoma to the pooled adenocarcinoma tissue (CA). Also, our results illustrated in figures $2 \mathrm{~A}$ and $2 \mathrm{C}$ are consistent with the previous study that were observed in ovarian tumor progression [23]. This result suggests that mitomiR-181 could be involved in tumor progression.

Notably, mitomiR-21 expression levels increased 2.5-folds $(p<0.005)$ in villous adenoma tissues when compared to tubular, tubulovillous and pooled adenocarcinoma (CA). Figures $3 \mathrm{~A}$ and $3 \mathrm{~B}$ showed markedly increased colorectal villous adenomas only compared to tubular, tubulovillous, adenocarcinomas and their normal adjacent tissues respectively. While progressively increased of mitomiR-21 expression levels from tubular adenoma to the pooled adenocarcinoma (CA) within individual patient samples with stage II of CRC tumors (Figure 3C).

As shown in Figure 4A, mitomiR-210 has a low level of expressions in precancerous tissues of tubular and tubulovillous adenomas when compared to villous adenoma. Moreover, approximately 6 -folds $(p<0.001)$ of mitomiR-210 expression levels were observed in adenocarcinoma (CA) when compared to tubular adenomas in the pooled sample tissues. Within the individual patient samples tissues with CRC stage I, the differential expression levels of mitomiR210 were high in villous adenocarcinoma and moderately increase in adenocarcinoma (CA) in the CRC stage II patient tissue samples (Figures 4B and 4C).

Noteworthy, mitomiR-378 has an appreciably progressive 
increased in the level of expressions from precancerous of tubular adenomas to adenocarcinoma $(\mathrm{CA})(p=0.005)$ as shown in figures $5 \mathrm{~A}, \mathrm{~B}, \mathrm{C}$.

Given all the studied mitomiRNAs in this study, mitomiR-

A)

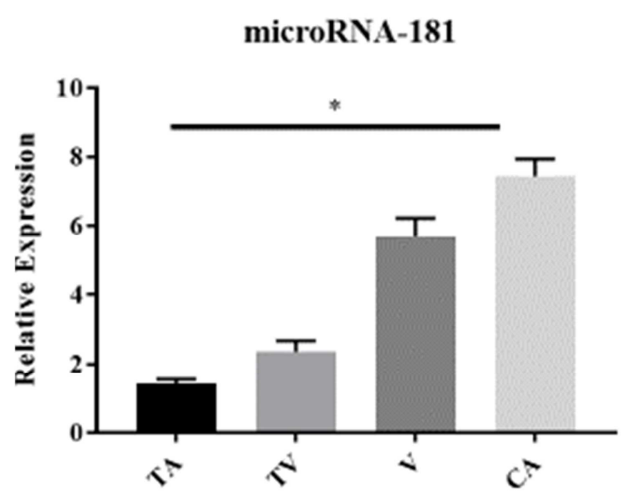

21 expression levels were the only to decline in the pooled adenocarcinoma tissues (Figure 3A). It suggests the down regulation of mitomiR-21 was becoming more stable in colorectal adenocarcinoma stages.

B)

Patient 1 mi181 Expression

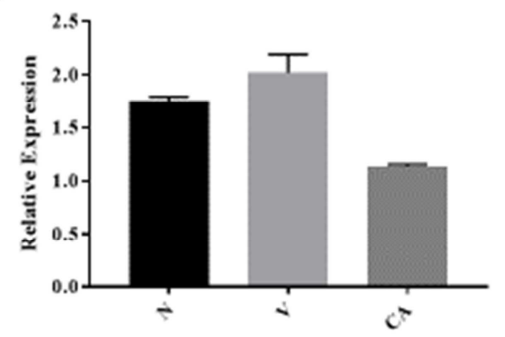

C)

Patient 2 mi181 Expression

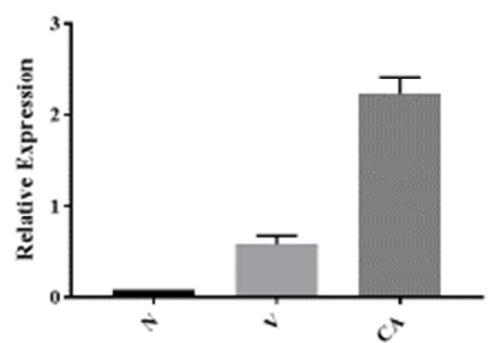

Figure 2. The relative expression levels of MitomiRNA-181 observed among patient tissue samples ( $n=64$ pairs) of colorectal adenomas (tubular (T), tubulovillous (TV), villous (V)), pooled adenocarcinoma (CA) and normal adjacent tissues (N). A) Correlation of each clinicopathological features of colorectal adenomas and adenocarcinoma tissues with expression levels of mitomiRNA-181 in extracted mitochondria RNA. Mean expression values from each tissue histological type were pooled and normalized to their paired normal adjacent tissues. Values are presented as relative expressions of mitomiRNA-181 which showed markedly increased progressively from colorectal adenomas to the pooled adenocarcinomas. B) Comparison of mitomiRNA-181 expression patterns in normal, villous, and adenoma tissues based on individual patient. Patient one is defined as 77 years old white female with stage I colon cancer. C) Comparison of mitomiRNA-181 expression patterns in 89 years old white female with stage II colon cancer. Bar diagrams show mean \pm from pooled results. Asterisk (*) $P<0.005$.

B)

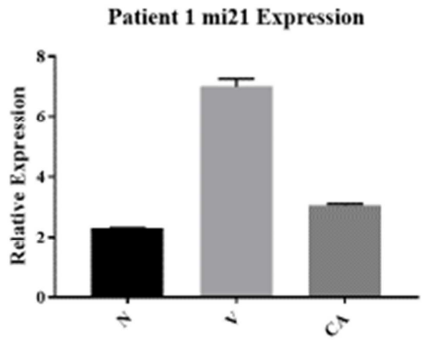

C)

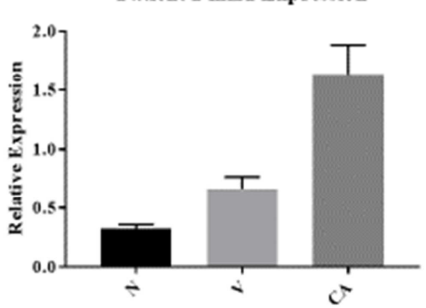

Figure 3. The relative expression levels of MitomiRNA-21 observed among patient tissue samples ( $n=64$ pairs) of colorectal adenomas: (tubular (T), tubulovillous (TV), villous (V)), pooled adenocarcinoma (CA) and normal adjacent tissues (N). A) Correlation of each clinicopathological features of colorectal adenomas and adenocarcinoma tissues with expression levels of mitomiRNA-21 in extracted mitochondria RNA. Mean expression values from each tissue histological type were pooled and normalized to their paired normal adjacent tissues. Values are presented as relative expressions of mitomiRNA-21 which showed markedly increased colorectal villous adenomas only compared to tubular, tubulovillous and pooled adenocarcinomas. B) Comparison of mitomiRNA-21 expression patterns in normal, villous and adenoma tissues based on individual patient. Patient one is defined as 77 years old white female with stage I colon cancer. C) Comparison of mitomiR expression patterns in 89 years old white female with stage II colon cancer. Bar diagrams show mean \pm from pooled results. Asterisk ("*) $P<0.001$. 
B)

A)

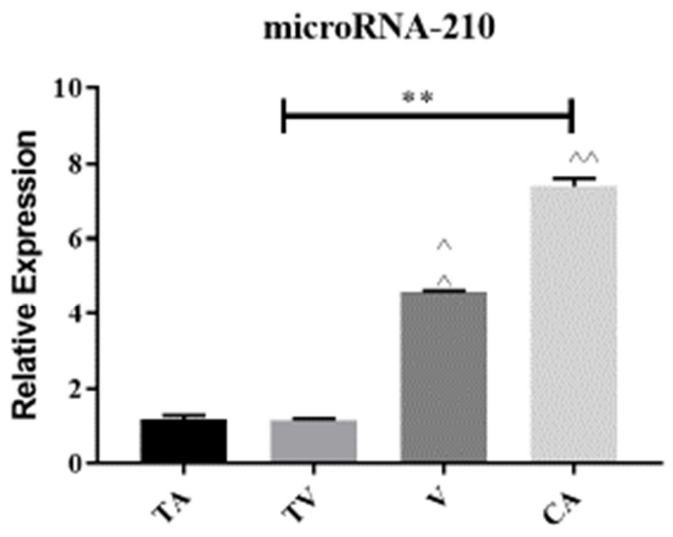

Patient 1 mi210 Expression

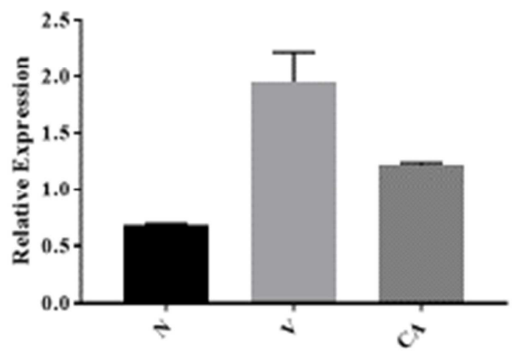

C)

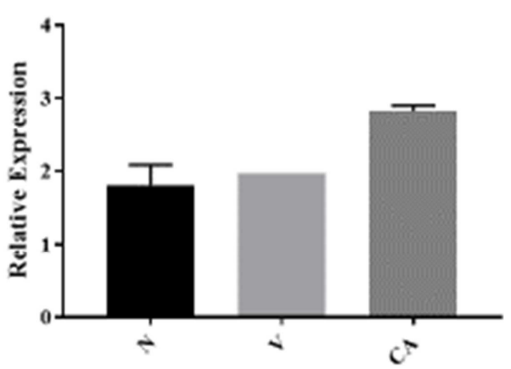

Figure 4. The relative expression levels of MitomiRNA-210 observed among patient tissue samples ( $n=64$ pairs) of colorectal adenomas, (tubular (T), tubulovillous (TV), villous (V)), to the pooled adenocarcinoma (CA) and normal surrounding tissues (N). A) Correlation of each clinicopathological features of colorectal adenomas and adenocarcinoma tissues with expression levels of mitomiRNA-210 in extracted mitochondria RNA. Mean expression values form each tissue histological type were pooled and normalized to their paired normal adjacent tissues. Values are presented as relative expressions of mitomiRNA-210 which showed markedly increased progressively from colorectal adenomas to adenocarcinomas. B) Comparison of mitomiRNA-210 expression patterns in normal, villous, and adenoma tissues based on individual patient. Patient one is defined as 77 years old white female with stage I colon cancer. C) Comparison of mitomiRNA-210 expression patterns in 89 years old white female with stage II colon cancer. Bar diagrams show mean \pm from pooled results. Asterisk ("*) $P<0.001$.

Patient 1 miR-378-5p
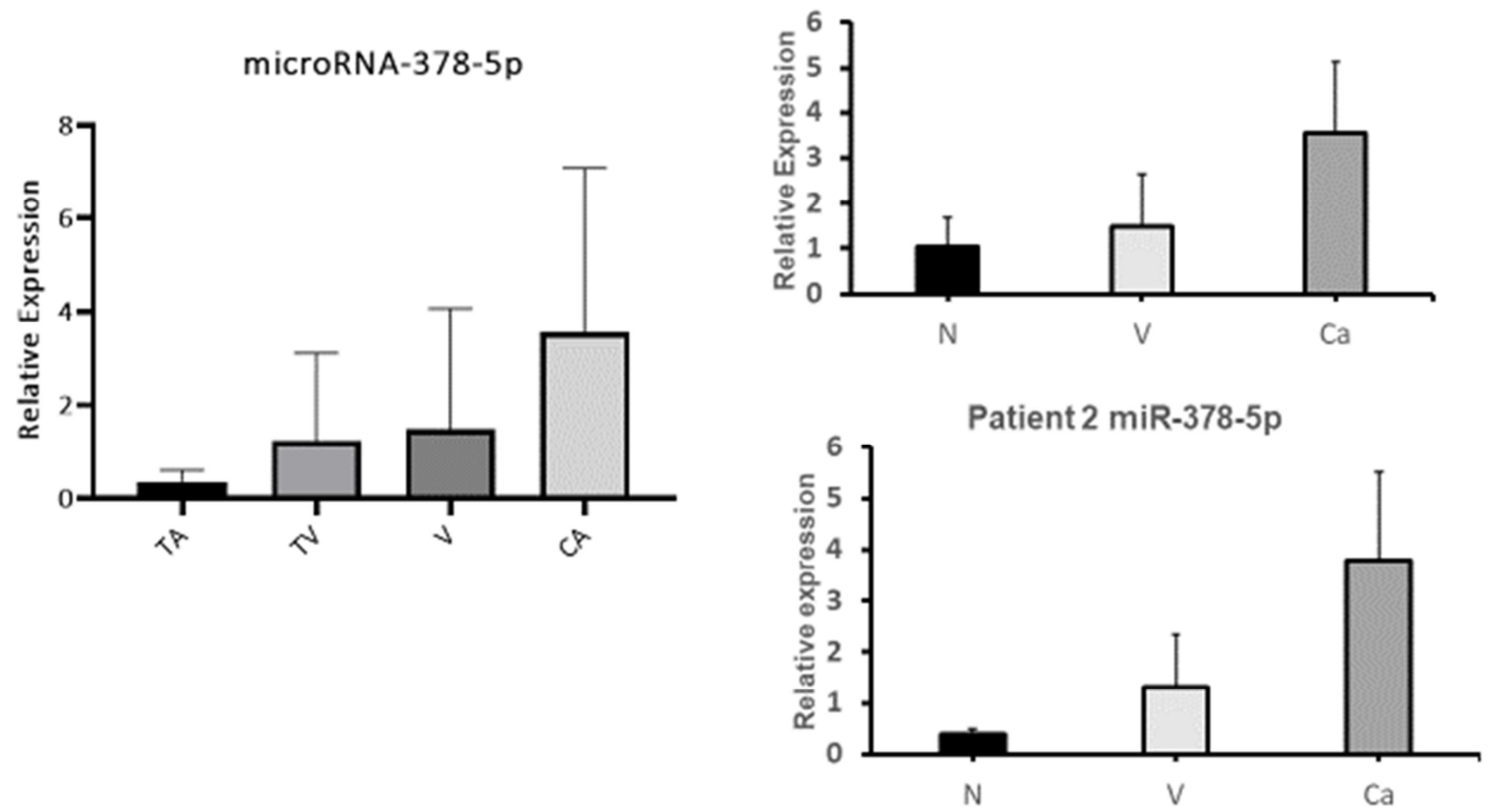

Figure 5. The relative expression levels of MitomiRNA-378 observed among patient tissue samples ( $n=64$ pairs) of colorectal adenomas. (tubular (T), tubulovillous (TV), villous (V)), to the pooled adenocarcinoma (CA) and normal adjacent tissues (N). A) Correlation of each clinicopathological features of colorectal adenomas and adenocarcinoma tissues with expression levels of mitomiRNA-378 in extracted mitochondria RNA. Mean expression values from each tissue histological type were pooled and normalized to their paired normal adjacent tissue. Values are presented as relative expressions of mitomiRNA-378 which showed markedly increased progressively from the colorectal adenomas to the pooled adenocarcinomas. B) Comparison of mitomiRNA-378 expression patterns in normal, villous, and adenoma tissues based on individual patient. Patient one is defined as 77 years old white female with stage I colon cancer. C) Comparison of mitomiRNA-378 expression patterns in 89 years old white female with stage II colon cancer. 


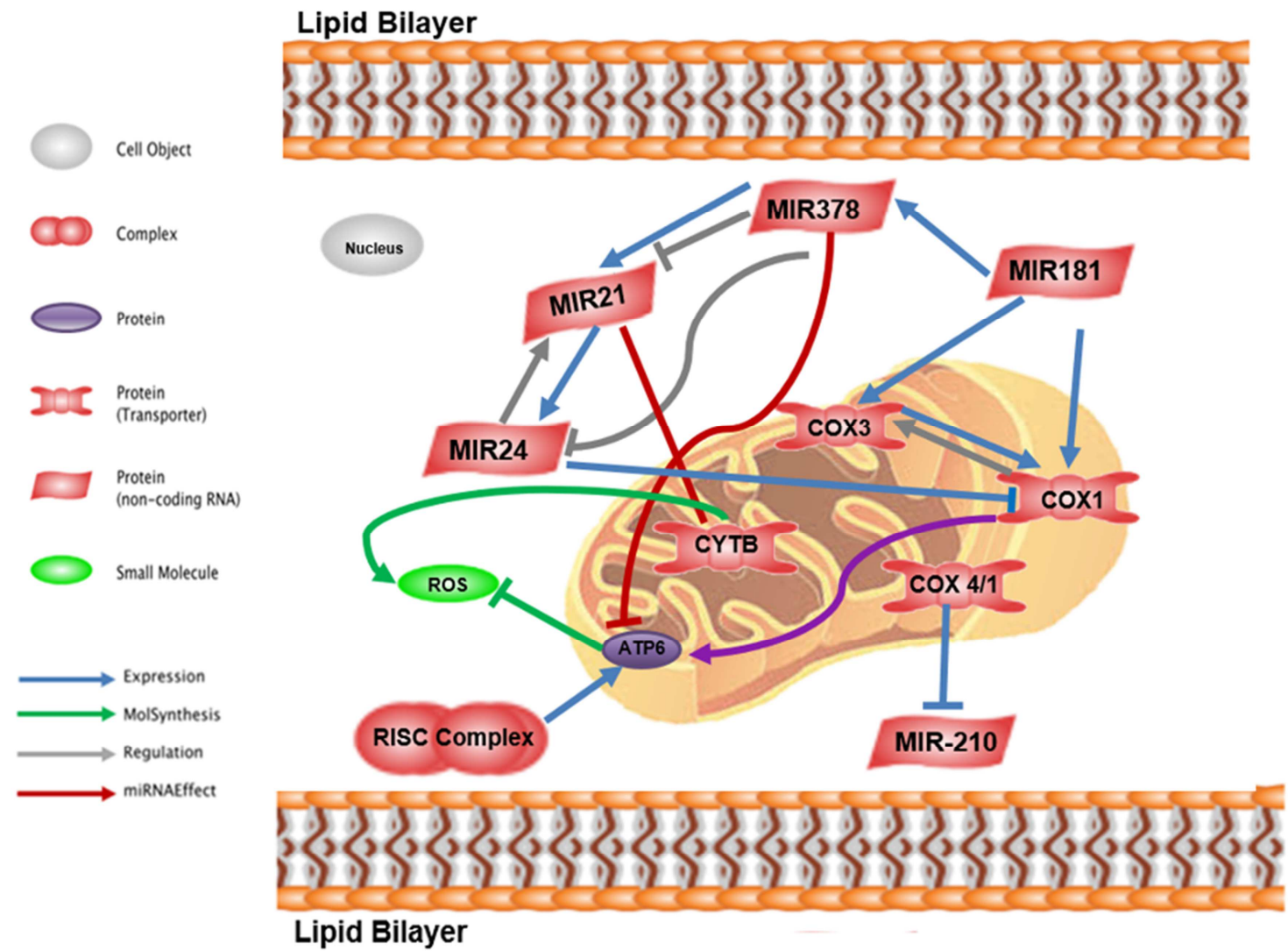

Figure 6. Schematic of mitomiRNAs interactions with mitochondrial encoded genes either positive or negative suggestively. Image was created using the Pathway Studio program and arrows indicate flow through signaling pathways; touching boxes indicate direct binding of mitomiRNAs signaling components. Data from various articles has shown that microRNAs have both positive and negative roles on subunits involved in oxidative phosphorylation. Within this interaction, reactive oxygen species may also play a role on the ability for mentioned microRNAs classification as an oncomiR or tumor suppressor.

\section{Discussion}

MitomiRs have the potential to play essential roles in cell processes such as proliferation, differentiation, and metastasis. MicroRNAs (miRs) have emerged as key regulators in gene expression targeting almost $30 \%$ of the human genome [24-26]. Given that the cell houses another genome aside from the nuclear genome many have speculated that microRNAs have been encoded or in part imported into the mitochondrial genome. This phenomenon provides a great avenue for therapeutic and biomarker discovery due to microRNAs roles in many tumor related diseases such as CRC. Ma et al. demonstrated that miR-34a expressions increased in CRC paraneoplastic tissues when compared to cancer tissues [27]. Based on these observed differences in the expression levels, it was suggested that miR-34a was more of a tumor suppressor gene in CRC. Perhaps, it could be speculated that microRNAs play various roles in CRC pathogenesis, as well as in other cancer tissue types. Past studies have associated mtDNA mutations with CRC adenomas development and progression [18, 19, 28]. It has been suggested that there are higher rates of mtDNA mutations compared to nuclear DNA that were resulting from ROS production during oxidative phosphorylation. Furthermore, an increased measurement of carbonyl contents as hallmark of oxidative stress have been involved in colorectal tumor progression [29]. Pathogenesis of other several age-related disorders, such as metabolic syndrome, macular degeneration, and neurodegenerative diseases have also been associated with oxidative stress [30]. A past study had suggested that some of the pre-miRNA's sequences seem to be processed in the mitochondria and aid in synthesis of mature miRNAs. Also, these pre-miRNAs could be immediately activated the mitochondrial transcripts or exported to the cytosol to be involved in mRNA-protein functions [31].

In this study, expressions of some microRNAs of mitochondrial-miRs (denoted as mitomiRs); miR-24, miR181, miR-210, miR-21 and miR378 were examined in different CRC tissue types due their previous involvement either inducing, inhibiting, or as a target of ROS productions $[32,33]$. Indeed, miRNA-24 has been shown to function as a tumor suppressive gene playing an important role in human cancer and other diseases [25]. However, other reports indicated that miR-24 was more oncogenic promoting cell proliferation and inhibiting apoptosis when over expressed in breast cancer cell lines MDA-MB-435A and MDA-MB-468 [34]. Also, expression levels of miR-24 were found to be elevated in breast cancer tissues when compared to normal adjacent tissues [33]. Whilst expressions of miR-24 roles were controversial among cancer cell type studies, our results 
showed that mitomiR24 progressively increased in the expression levels among precancerous of CRC adenoma to invasive adenocarcinoma tissues (Figure 1A). In general, our results appeared to be similar with previously reported miR24 study in CRC tissues [20]. It is noteworthy that analysis of the individual patients we observed different levels of mitomiR-24 expressions that depend on various degrees of tumor contents in the tissues and the stages of each patient tissue as shown in Figures $1 \mathrm{~B}$ and $\mathrm{C}$. The results suggest that the tumor microenvironment could be playing a huge role in these observed mitomiR-24 expressions difference between the two patients with different stages and tumor contents. These results could be insightful when proposing personalized medicine initiative.

In this study, mitomiRs-181 expressions showed an appreciably progressive increased from colorectal tubular adenoma to adenocarcinoma samples but not in stage I individual sample. Parikh et al. [26] previous study has suggested that miR-181 plays an oncogenic role in ovarian cancer cells survival, migration, drug resistance, and invasion by inducing TGF- $\beta$ that target SMAD7. The process resulted to epithelial-mesenchymal transition in epithelial ovarian cancer cells and tumors. Also studies with cervical cancer cells, miR-181 inhibition led to increased apoptosis and cell cycle arrest [35]. Moreover, high miR-181 expression levels had been attributed to liver metastasis, and inducing EMT in prostate cancer [36, 37]. The progressively elevated mitomiR-181 expression levels from early CRC adenoma of tubular to pooled invasive adenocarcinomas observed in this study indicates that mitomiR-181 expressions acting as an oncogene that could positively influenced the progression of colorectal tumor of adenomas to invasive adenocarcinomas.

We also examined the mitomiR-21, 210 and 378 expressions and their association with CRC patient tissues. We found that mitomiR-21 expression was elevated in both aggregated tissue samples of CRC villous adenomas and in stage I individual patient sample (Figures $3 \mathrm{~A}$ and $\mathrm{B}$ ). In addition, mitomiR-21 progressively increased in $\mathrm{CRC}$ villous and adenocarcinoma in stage II with different contents of tumors (Figure C). Furthermore, we observed mitomiR-210 and 378 expressions were elevated progressively in both in patient aggregated and individuals of stages I and II with different tumor contents from early CRC adenomas to invasive adenocarcinomas. The exception was the mitomiR 210 expression was relatively elevated in villous adenoma of individual with stage I (Figure 4B).

Even though the mitochondrial genome is only composed of 13 genes, there are over 2000 proteins that have been identified that participated in the overall functions of the mitochondria $[38,39]$. Past studies suggest that miRNAs of mitochondrial origin can regulate nuclear mRNA translation that alter cell phenotype [40-42]. Hence, elucidating the crosstalk mechanism between the nuclear and mitochondrial genome regarding the functional impacts of miRNAs in tumor progressive stages and possible ROS productions, (as shown in Figure 6), will pivotally aid in the clinicopathological characteristics of CRC. Our lab had reported a decreased in mitochondrial isolated COXIV-1 a nuclear encoded subunit of complex IV with the progressive stages of CRC adenomas [43]. This finding was strikingly important given the role of COXIV-1 in regulating of the mitochondrial encoded subunits of complexes IV and V, as

well as regulation of oxidative phosphorylation. Additionally, in rat cardiomyocytes depressed COXIV-1 expression led to modulated ATP production [44]. Moreover, COXIV-1 and COX10 another subunit of complex IV is also a proposed target of miRNA-210. COX10 has been shown to be involved in down-regulation of colorectal cancer cells in response to induction of miR-210 [14].

As a potential mitomiR target, it is important to mention that miR-210 is associated with ROS and is known to be induced by hypoxia. Past studies indicate that the expressions of miR-210 in colorectal tumors have a positive correlation with hypoxic marker, carbonic anhydrate [15]. Interestingly, it has been previously shown that miR-210 was upregulated in colorectal cancer when compared with normal adjacent tissues which is consistent with our results [45]. Studies indicate that miR-210 expressions in breast cancer, non-small cell lung cancer, glioma, and clear cell renal cancer were relatively elevated. Also, meta-analysis shown elevated miR210 expressions were significantly correlated to low survival rates in patients with different cancers [46].

Studies have shown that nuclear miRs-21 were considered as oncogenic that are generally upregulated in many cancer cells [47, 48]. Other studies using epithelial ovarian cancer indicated that miRs-21 were overexpressed in tissues and cell lines by knocked down using an inhibitor shown a significant reduction in cell proliferation and a decreased in cell invasions and migrations [47]. Although targets of miR-21 include PTEN, PDCD4, BTG2, and MKK3 tumor suppressor proteins creating both pro-survival and anti-apoptotic properties for this microRNA [32]. Its biological functions in the development in CRC progression remains unclear. In our study, we found mitomiR-21overexpressions in CRC villous adenomas in both aggregated and individual stage I tissue samples but repressed invasive adenocarcinoma. The overexpression of mitomiR-2 1 in early CRC villous adenoma could be inferred as linkage between the larger polyps growing rapidly and mitochondrial encoded microRNA increases during the progression of carcinogenesis, suggesting the coordination of mitomiR-21 role in reprogramming of CRC tumor metabolism [49].

There are several studies that focused nuclear encoded miRs-378 functions as a tumor suppressor that plays an important role in inhibiting tumor growth and invasion and that reverse drug resistance to cisplatin in lung cancer [50]. Previous study demonstrated a decreased miR-378 expression levels in gliomas with high tumor invasiveness and poor prognosis [51, 52]. Moreover, Zeng et al., [33] indicated that miR-378 expression alleviated in the malignant phenotypes of colon cancer cells by inhibiting the Wnt/ $\beta$-catenin pathway, which appears to be upregulated in the cancer process. This pathway is closely associated with both chronic inflammation and oxidative stress in cancers. However, the 
role of elevated expression of mitochondrial encoded, mitomiR-378 in CRC early colorectal tumor and adenocarcinoma remains to be elucidated. In this study, it was found that mitomiR-378 expressions progressively increased from precancerous of tubular adenoma to invasive adenocarcinoma, and in both aggregated and individual stages I and II suggesting that mitochondrial derived mitomiR-378 positively influence CRC tumoriogenesis. Therefore, the role of mitomiRs in the development, progression of CRC needs to be further investigated in future studies.

\section{Conclusion}

Based on this study, our data indicate that miRNAs isolated from mitochondrial (mitomiRs) are differentially expressed among precancerous of CRC adenoma and adenocarcinoma tissues. Moreover, our study for the first time demonstrated higher elevation of mitomiRs expression levels in CRC tissues of adenocarcinoma (CA) compared with precancerous of polyps (TA, TV, V). These data collectively suggest that mitomiRs, particularly mitomiR-378 expressions may play an important role in the transformation of CRC. Our findings contribute to the current understanding of the miRs derived from mitochondrial in the procedural of CRC progression, which warrants further investigation in the regulate of mitochondrial functionalities leading to a more favorable environment for transformation or progression of colorectal adenomatous polyps to adenocarcinomas.

\section{Author Contributions}

L. M. and F. A., conceived and designed the study. L. M., F. A., S. B. and K. A., coordinated the data collection. L. M. and F. A were responsible for the data analysis. F. A., L. M., K. A., K. P and X. Y. interpreted the results. F. A., L. M. and M. P, drafted the manuscript. All authors reviewed and approved the final version of the article.

\section{List of Abbreviations}

$\begin{array}{ll}\text { AA } & \text { African American } \\ \text { AJCC } & \text { American Joint Committee on Cancer } \\ \text { CRC } & \text { Colorectal Cancer } \\ \text { CAU } & \text { Caucasian } \\ \text { miRNAs } & \text { MicroRNAs } \\ \text { MitomiRNAs } & \text { Mitochondrial MicroRNAs } \\ \text { TA } & \text { Tubular Adenoma } \\ \text { TV } & \text { Tubulovillous Adenoma } \\ \text { V } & \text { Villous Adenoma } \\ \text { CA } & \text { Adenocarcinoma } \\ \text { ROS } & \text { Reactive oxygen species }\end{array}$

\section{Conflicts of Interest}

The authors declare no conflict of interest.

\section{Acknowledgements}

NIH-NIGMS (GM122669 and GM099663) funds awarded to Dr. Felix O Aikhionbare supported this research.

\section{References}

[1] Rawla, P.; Sunkara, T.; Barsouk, A. Epidemiology of colorectal cancer: incidence, mortality, survival, and risk factors. Prz Gastroenterol. 2019, 14 (2): 89-103.

[2] Bujanda, L.; Cosme, A.; Gil I.; Juan, I.; Arenas-Mirave JI, Malignant colorectal polyps. World J Gastroenterol. 2010; 16, (25).

[3] Munteanu, I.; Mastalier B. Genetics of colorectal cancer. $J$ Med Life. 2014; 7 (4), 507-511.

[4] Sun, D.; Yu, F.; Ma, Y.; Zhao, R.; Chen, X.; Zhu, J.; Zhang, CY.; Chen, J. Z; H Hang, J. MicroRNA-31 activates the RAS pathway and functions as an oncogenic MicroRNA in human colorectal cancer by repressing RAS p21 GTPase activating protein 1 (RASA1). The Journal of biological chemistry. 2013 , 288 (13), 9508-9518.

[5] Kent, O.; Mendell, J.; Rottapel, R. Transcriptional Regulation of miR-31 by Oncogenic KRAS Mediates Metastatic Phenotypes by Repressing RASA1. Molecular Cancer Research: MCR 2016, 14 (3), 267-277.

[6] Catalanotto C.; Cogoni C.; Zardo G. MicroRNA in Control of Gene Expression: An Overview of Nuclear Functions. Int $J$ Mol Sci. 2016, 17 (10): 1712.

[7] Boeckx N.; Op de Beeck K.; Beyens M.; Deschoolmeester V.; Hermans C.; De Clercq P.; Garrigou S.; Normand C.; Monsaert E.; Papadimitriou K et al. Mutation and Methylation Analysis of Circulating Tumor DNA Can Be Used for Followup of Metastatic Colorectal Cancer Patients. Clinical Colorectal Cancer 2018. 17 (2), e369-e379.

[8] Bienertova-Vasku J.; Sana J.; Slaby O. The role of microRNAs in mitochondria in cancer. Cancer Letters. 2013, $336(1), 1-7$.

[9] Strubberg A.; Madison B. MicroRNAs in the etiology of colorectal cancer: pathways and clinical implications. Disease Models \& Mechanisms. 2017, 10 (3): 197-214.

[10] Clancy C.; Joyce M.; Kerin M. The use of circulating microRNAs as diagnostic biomarkers in colorectal cancer. Cancer Biomarkers. 2015, 15 (2): 103-113.

[11] Kim N.; Cha Y.; Kang S.; Lee Y.; Lee I.; Cha S.; Ryu J.; Na J.; Park C.; Yoon H.; et al. p53 reg $\mu$ lates nuclear GSK-3 levels through miR-34-mediated Axin2 suppression in colorectal cancer cells. Cell Cycle. 2013, 12 (10): 1578-1587.

[12] Nagel R.; le Sage C.; Diosdado B.; van der Waal M..; Oude Vrielink J.; Bolijn A.; Meijer G.; Agami R. Regulation of the Adenomatous Polyposis Coli Gene by the miR-135 Family in Colorectal Cancer. Cancer Research. 2008, 68 (14): 5795 .

[13] Yang J.; Ma D.; Fesler A.; Zhai H.; Leamniramit A.; Li W.; Wu S.; Ju J. Expression analysis of microRNA as prognostic biomarkers in colorectal cancer. Oncotarget. 2017, 8 (32): 52403-52412. 
[14] Chen Z.; Li Y.; Zhang H.; Huang P.; Luthra R. Hypoxiareg $\mu$ lated microRNA-210 mod $\mu$ lates mitochondrial function and decreases ISCU and COX10 expression. Oncogene. 2010, 29 (30): 4362-4368.

[15] Nijhuis A.; Thompson H.; Adam J.; Parker A.; Gammon L.; Lewis A.; Bundy J.; Soga T.; Jalaly A.; Propper D.; et al. Remodelling of microRNAs in colorectal cancer by hypoxia alters metabolism profiles and 5 fluorouracil resistance. Human Molecular Genetics. 2017, 26 (8): 1552-1564.

[16] Thulasingam S.; Massilamany C.; Gangaplara A.; Dai H.; Yarbaeva S.; Subramaniam S.; Riethoven J.; Eudy J.; Lou M.; Reddy J. miR-27b*, an oxidative stress-responsive microRNA modulates nuclear factor-kB pathway in RAW 264.7 cells. Molecular and Cellular Biochemistry. 2011, 352 (1-2): 181188.

[17] Shaughnessy D.; McAllister K.; Worth L.; Haugen A.; Meyer J.; Domann F.; Van H.; Mostoslavsky R.; Bultman S.; Baccarelli A.; et al. Mitochondria, energetics, epigenetics, and cellular responses to stress. Environ Health Perspect. 2014, 122 (12): 1271-1278.

[18] Adams G.; Mehrabi S.; Vatcharapijarn Y,; Iyamu O.; Akwe J.; Grizzle W.; Yao X.; Aikhionbare F. Frequencies of mtDNA mutations in primary tissue of colorectal adenopolyps. Frontiers in Bioscience (Elite edition). 2013, 5: 809-813.

[19] Aikhionbare F.; Khan M.; Carey D.; Okoli J.; Go R. Is cumplative frequency of mitochondrial DNA variants a biomarker for colorectal tumor progression? Molecular Cancer. 2004, 3: 30-30.

[20] Eslamizadeh S.; Heidari M.; Agah S; Faghihloo E.; Ghazi H.; Mirzaei A.; Akbari A.; The role of microRNA signature as diagnostic biom

[21] Raisch J.; Darfeuille-Michaud A.; Nguyen H. Role of microRNAs in the immune system, inflammation and cancer. World Journal of Gastroenterology: WJG. 2013, 19 (20): 2985-2996.

[22] Mingxi Z.; Linlin Z.; Liangping L.; Kang C. miR-378 suppresses the proliferation, migration and invasion of colon cancer cells by inhibiting SDAD1. Cellular \& Molecular Biology Letters. 2017, 22 (1): 12.

[23] Peltier HJ.; Latham GJ. Normalization of microRNA expression levels in quantitative RT-PCR assays: identification of suitable reference RNA targets in normal and cancerous human solid tissues. RNA. 2008; 14 (5): 844-852.

[24] Wallace L; Mehrabi S.; Bacanamwo M.; Yao X.; Aikhionbare F. Expression of mitochondrial genes MT-ND1, MT-ND6, MT-CYB, MT-COI, MT-ATP6, and 12S/MT-RNR1 in colorectal adenopolyps. Tumour biology: the journal of the International Society for Oncodevelopmental Biology and Medicine. 2016, 37 (9): 12465-12475

[25] Guo Y.; Fu W.; Chen H.; Shang C.; Zhong M. miR-24 functions as a tumor suppressor in Hep2 laryngeal carcinoma cells partly through down-reg $\mu$ lation of the S100A8 protein. Oncology Reports. 2012, 27 (4): 1097-1103.

[26] Parikh A.; Lee C.; Joseph P.; Marchini S.; Baccarini A.; Kolev V.; Romualdi C.; Fruscio R.; Shah H.; Wang F.; et al. microRNA-181a has a critical role in ovarian cancer progression through the regulation of the epithelialmesenchymal transition. Nature Communications. 2014, 5: 2977.
[27] Ma Z.; Kong X.; Cui G.; Ren C.; Zhang Y.; Fan S.; Li Y. Expression and clinical significance of miRNA-34a in colorectal cancer. Asian Pacific Journal of Cancer Prevention: APJCP. 2014, 15 (21): 9265-9270.

[28] Mehrabi S.; Akwe J.; Adams G.; Grizzle W.; Yao X.; Aikhionbare F. Analysis of mtDNA sequence variants in colorectal adenomatous polyps. Diagn Pathol. 2010, 5 (1): 66.

[29] Mehrabi S.; Wallace L.; Cohen S.; Yao X.; Aikhionbare F. Differential Measurements of Oxidatively Modified Proteins in Colorectal Adenopolyps. Int J Clin Med. 2015, 6 (4): 288-299.

[30] Liguori, I., Russo, G., Curcio, F., Bulli, G., Aran, L., DellaMorte, D., Gargiulo, G., Testa, G., Cacciatore, F., Bonaduce, D., Abete, P. Oxidative stress, aging, and diseases. Clinical Interventions in Aging. 2018, 13: 757-772.

[31] Bandiera S.; Matégot R.; Girard M., Demongeot J.; HenrionCaude A. MitomiRs delineating the intracellular localization of microRNAs at mitochondria. Free Radical Biology and Medicine. 2013, 64: 12-19.

[32] He J.; Jiang B.; Interplay Between Reactive Oxygen Species and MicroRNAs in Cancer. Current Pharmacology Reports. 2016, 2 (2): 82-90.

[33] Zeng M.; Zhu L.; Li L.; Kang C. miR-378 suppresses the proliferation, migration and invasion of colon cancer cells by inhibiting SDAD1. Cellular \& Molecular Biology Letters. 2017, 22 (1): 12.

[34] Lu K.; Wang J.; Song Y.; Zhao S.; Liu H.; Tang D.; Pan B.; Zhao H.; Zhang Q. miRNA-24-3p promotes cell proliferation and inhibits apoptosis in human breast cancer by targeting p27Kip1. Oncology Reports. 2015, 34 (2): 995-1002.

[35] Xu H.; Zhu J.; Hu C.; Song H.; Li Y. Inhibition of microRNA181a may suppress proliferation and invasion and promote apoptosis of cervical cancer cells through the PTEN/Akt/FOXO1 pathway. Journal of Physiology and Biochemistry. 2016, 72 (4): 721-732.

[36] Tong S.; Liu J.; Wang X.; Qu L. microRNA-181 promotes prostate cancer cell proliferation by regulating DAX-1 expression. Experimental and Therapeutic Medicine. 2014, 8 (4): $1296-1300$.

[37] Ji D.; Chen Z,; Li M.; Zhan T.; Yao Y.; Zhang Z.; Xi J.; Yan L.; $\mathrm{Gu}$ J. MicroRNA-181a promotes tumor growth and liver metastasis in colorectal cancer by targeting the tumor suppressor WIF-1. Molecular Cancer. 2014, 13 (1): 86.

[38] Choudhury A.; Singh K.; Mitochondrial determinants of cancer health disparities. Seminars in Cancer Biology. 2017, 47: 125-146.

[39] Wei Z.; Su W.; Lou H.; Duan S.; Chen G. Trafficking pathway between plasma membrane and mitochondria via clathrinmediated endocytosis. Journal of Molecular Cell Biology. 2018, 10 (6), 539-548.

[40] Cui Y.; Wang Y.; Liu M.; Qiu L.; Xing P.; Wang X.; Ying G.; $\mathrm{Li}$ B. Determination of glucose deficiency-induced cell death by mitochondrial ATP generation-driven proton homeostasis. $J$ Mol Cell Biol. 2017, 9 (5): 395-408.

[41] Kwak S.; Yoo J.; An H.; Bae I.; Park M.; Kim J.; Han Y. miR5003-3p promotes epithelial-mesenchymal transition in breast cancer cells through Snail stabilization and direct targeting of E-cadherin. J Mol Cell Biol. 2016, 8 (5): 372-383. 
[42] Duarte F.; Palmeira C.; Rolo A. The Role of microRNAs in Mitochondria: Small Players Acting Wide. Genes (Basel). 2014, 5 (4): 865-886

[43] Wallace L.; Cherian A.; Adamson P.; Bari S.; Banerjee S.; Flood M.; Simien M.; Yao X.; Aikhionbare F. Comparison of Pre- and Post-translational Expressions of COXIV-1 and MTATPase 6 Genes in Colorectal Adenoma-Carcinoma Tissues. $J$ Carcinog Mutagen. 2018, 9 (2): 319.

[44] Zhang X.; Sch $\mu$ lze P. MicroRNAs in heart failure: Non-coding reg $\mu$ lators of metabolic function. Biochimica et Biophysica Acta. 2016, 1862 (12): 2276-2287.

[45] Qu A.; Du L.; Yang Y.; Liu H.; Li J.; Wang L.; Liu Y.; Dong Z.; Zhang X.; Jiang X.; et al. Hypoxia-Inducible MiR-210 Is an Independent Prognostic Factor and Contributes to Metastasis in Colorectal Cancer. PLoS ONE. 2014, 9 (3): e90952.

[46] Xie X.; Wu W.; Liang L.; Han S.; Chen T.; Pan S.; Xue M.; Li S. Prognostic role of microRNA-210 in various carcinomas: a meta-analysis. International Journal of Clinical and Experimental Medicine. 2015, 8 (9): 15283-15289.

[47] Huang Z.; Huang D.; Ni S.; Peng Z.; Sheng W.; Du X. Plasma
microRNAs are promising novel biomarkers for early detection of colorectal cancer. International Journal of Cancer. 2010, 127 (1): 118-126.

[48] Pfeffer S.; Yang C.; Pfeffer L. The Role of miR - 21 in Cancer. Drug Development Research. 2015, 76 (6): 270-277.

[49] Wu C.; Ng S.; Dong Y.; Ng S.; Leung W.; Lee C.; Wong Y.; Chan F.; Yu J.; Sung J. Detection of miR-92a and miR-21 in stool samples as potential screening biomarkers for colorectal cancer and polyps. Gut. 2012, 61 (5): 739.

[50] Chen X.; Jiang Y.; Huang Z.; Li D.; Chen X.; Cao M.; et al. miRNA-378 reverses chemoresistance to cisplatin in lung adenocarcinoma cells by targeting secreted clusterin. Sci Rep. 2016, 6: 19455 .

[51] Li B.; Wang Y.; Li S.; He H., Sun F.; Wang C.; et al. Decreased expression of miR-378 correlates with tumor invasiveness and poor prognosis of patients with glioma. Int $J$ Clin Exp Pathol. 2015, 8: 7016-21.

[52] Shukla G.; Singh J.; Barik S. MicroRNAs: Processing, Maturation, Target Recognition and Regulatory Functions. Mol Cell Pharmacol. 2011, 3 (3): 83-92. 